\title{
The Weakly Neighborly Polyhedral Maps on the 2-Manifold with Euler Characteristic -1
}

\author{
Amos Altshuler ${ }^{1}$ and Ulrich Brehm ${ }^{2}$ \\ Ben-Gurion University of the Negev, Beer-Sheva, Israel \\ ${ }^{2}$ FB Mathematik, Technische Universität Berlin, 1000 Berlin 12, FR Germany
}

\begin{abstract}
A weakly neighborly polyhedral map (w.n.p. map) is a two-dimensional cell-complex which decomposes a closed 2-manifold without boundary, such that for every two vertices there is a 2-cell containing them. We prove that there are just four w.n.p. maps with Euler characteristic -1 and we describe them.
\end{abstract}

\section{Introduction}

A weakly neighborly polyhedral map (w.n.p. map) is a two-dimensional topological cell-complex which decomposes a closed 2-manifold (usually without boundary), such that for every two vertices there is a 2 -cell containing them. The zero-, one-, and two-dimensional cells of the map are its vertices, edges, and facets, respectively.

Some aspects of w.n.p. maps of arbitrary genus have been studied in [4], where a detailed study of w.n.p. maps on the sphere is also given. (The genus of a 2-manifold is defined as $g=\frac{1}{2}(2-\chi)$, where $\chi$ denotes the Euler characteristic of the manifold. Thus the projective plane is of genus $\frac{1}{2}$, and the genus of the 2-manifold studied in the present paper is $1 \frac{1}{2}$.) In [5] a complete list of the five w.n.p. maps on the torus is given. In [1] we prove that there are no orientable w.n.p. maps of genus 2. Complete lists of the nonorientable w.n.p. maps with Euler characteristics 1, 0, and -2 are given in [3] and [2].

In the present paper we investigate the w.n.p. maps with Euler characteristic -1 (i.e., $g=1 \frac{1}{2}$ ). We prove the following:

Theorem. There are precisely four w.n.p. maps with Euler characteristic -1. They are the maps $\mathscr{A}, \mathscr{B}, \mathscr{C}$, and $\mathscr{D}$ depicted in Fig. 1.

The proof of our theorem is heavily based on some of the results obtained in [4]. We also refer the reader to [4] for motivation for the concept of the w.n.p. 


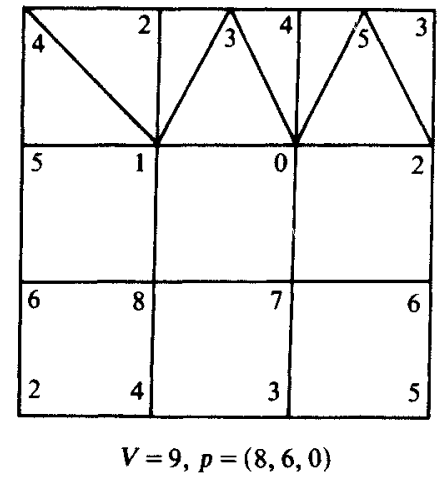

$\mathscr{A}$

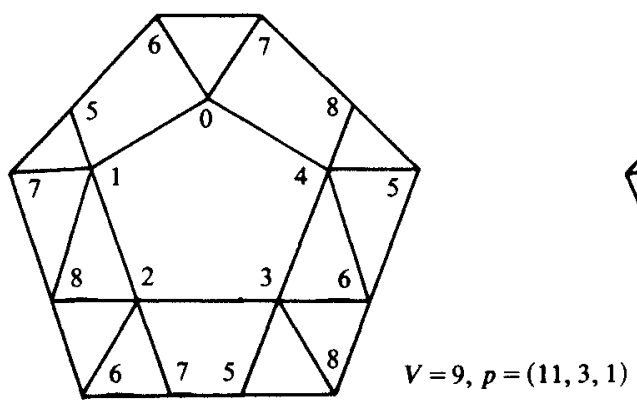

9

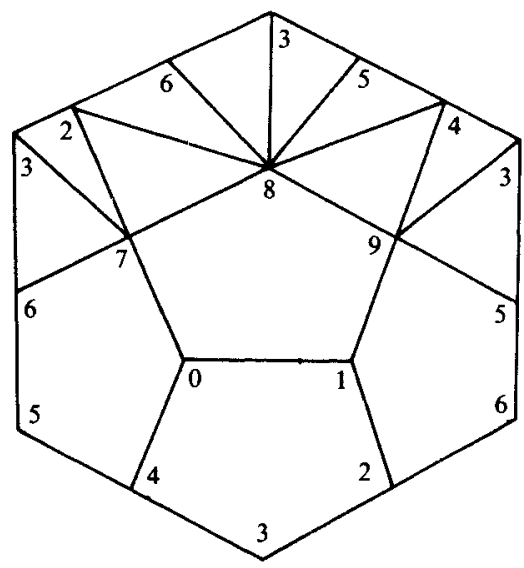

$V=10, p=(10,0,4)$

$\mathscr{D}$

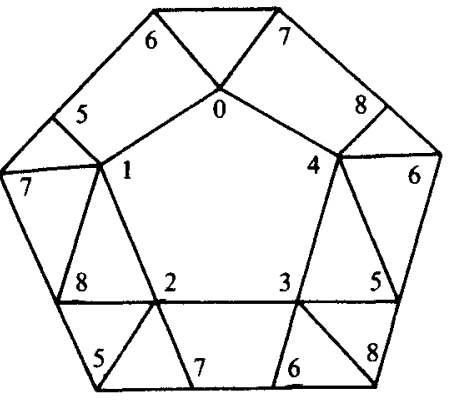

$\mathscr{C}$

Fig. 1

map. We prove there that the number of w.n.p. maps on any 2 -manifold $N$ other than the 2-sphere is finite, and we give an upper bound for the number of vertices in a w.n.p. map on $N$ as a function of the genus $g$ of $N$. For $g=1 \frac{1}{2}$ this upper bound is 11 .

The following will serve as our standard notation. We assume a polyhedral (or w.n.p.) map $M$ of genus $g=1 \frac{1}{2}$ with $V$ vertices $v_{1}, \ldots, v_{V}$ and $F$ facets $f_{1}, \ldots, f_{F} . k_{i}$ is the number of vertices of $f_{i}(1 \leq i \leq F)$, and we take the notation to be such that $k_{1} \geq k_{2} \geq \cdots \geq k_{F}$. The vertices, too, are labeled according to the decreasing order of valences (degrees), that is, $\operatorname{deg} v_{1} \geq \operatorname{deg} v_{2} \geq \cdots \geq \operatorname{deg} v_{v} \cdot p_{i}$ denotes the number of $i$-gonal facets of the map $M$ and $\left(p_{3}, p_{4}, \ldots\right)$ is the p-vector of $M . V_{i}$ denotes the number of vertices of valence $i$ and $\left(V_{3}, V_{4}, \ldots\right)$ is the $v$-vector of $M . N\left(f_{j}\right)$ is the set of facets sharing a common edge with the facet $f_{j}$ and $N\left(v_{j}\right)$ is the set of vertices joined by an edge to the vertex $v_{j}$. 
The proof of our theorem is given in Section 2 and consists of two parts. First we find a set of eight candidates for $p$-vectors (summarized in Table 1) such that every w.n.p. map with $g=1 \frac{1}{2}$ has its $p$-vector in this set. The second part consists of a detailed investigation of each of the eight candidates. In each of these cases we assume that there is some w.n.p. map realizing that "p-vector." In five of the eight cases we show that this leads to a contradiction, and in the remaining three cases we find all the possible maps.

In Section 3 we describe how the four maps mentioned in our theorem can be obtained from certain w.n.p. maps of lower genus, by using certain replacement transformations. We conclude by describing the symmetry groups of our four w.n.p. maps.

For the reader's convenience, we quote some of the results proved in [4] which will be used here, with their reference numbers. Those results are adapted here for $g=1 \frac{1}{2}$. They are as follows: $\left([x]\right.$ denotes the least integer $\geq x$, and $\left|f_{i} \cap f_{j}\right|$ denotes the number of vertices common to the facets $f_{i}, f_{j}$ ).

The following hold for every polyhedral map with $g=1 \frac{1}{2}$ :

$$
\begin{gathered}
2 V+2=\sum_{i=1}^{F}\left(k_{i}-2\right), \\
k_{i} \geq 3 \quad \text { for every } \quad 1 \leq i \leq F, \\
\sum_{i=2}^{1+\operatorname{deg} v_{i}}\left(\operatorname{deg} v_{i}-3\right)^{2}+\sum_{i=2+\operatorname{deg} v_{1}}^{v}\left(\operatorname{deg} v_{i}-2\right)^{2} \leq\left(F-\operatorname{deg} v_{1}\right)\left(F-\operatorname{deg} v_{1}-1\right) .
\end{gathered}
$$

The following hold for every w.n.p. map with $g=1 \frac{1}{2}$ :

$$
\begin{gathered}
V^{2}-7 V-6=\sum_{i=1}^{F}\left(k_{i}-2\right)\left(k_{i}-3\right), \\
k_{1}>\frac{1}{2} V-1, \\
\sum_{\left|f_{i} \cap f_{j}\right|=2} k_{i}-\sum_{f_{i} \cap f_{j}=\varnothing}\left(k_{i}-2\right)=\left(k_{j}-2\right)\left(V-k_{j}+2\right) \quad \text { for every } \quad 1 \leq j \leq F, \\
\sum_{f_{i} \in N\left(f_{j}\right)} k_{i} \geq\left(k_{j}-2\right)\left(V-k_{j}+2\right) \quad \text { for every } \quad 1 \leq j \leq F, \\
\sum_{i=2}^{k_{1}+1} k_{i} \geq\left(k_{1}-2\right)\left(V-k_{1}+2\right), \\
\sum_{i=2}^{k_{1}+1}\left(k_{i}-3\right)^{2}+\sum_{i=k_{1}+2}^{F}\left(k_{i}-2\right)^{2} \leq\left(V-k_{1}\right)\left(V-k_{1}-1\right), \\
\sum_{v_{i} \in N\left(v_{j}\right)}\left(\operatorname{deg} v_{i}-3\right)^{2}+\sum_{v_{j} \neq v_{i} \in N\left(v_{j}\right)}\left(\operatorname{deg} v_{i}-2\right)^{2} \\
\leq\left(F-\operatorname{deg} v_{j}\right)\left(F-\operatorname{deg} v_{j}-1\right), \quad \text { for every } 1 \leq j \leq V . \\
k_{1} \leq \max \left\{6, \frac{1}{2}(V+1)\right\} .
\end{gathered}
$$


From [5] we quote

$$
\begin{aligned}
& \qquad 6+\sum_{i}(6-i) P_{i}=2 \sum_{i}(i-3) V_{i} \\
& \text { for every polyhedral map with } g=1 \frac{1}{2} \text {. }
\end{aligned}
$$

Lemma 1. If $v$ is a vertex of valence l in a w.n.p. map $M$ and the I facets of $M$ incident to $v$ have $k_{i_{1}}, k_{i_{2}}, \ldots, k_{i_{1}}$ vertices, then $\sum_{j=1}^{l} k_{i_{1}}=V+2 l-1$.

Lemma 2. If $M$ is a polyhedral map and $f$ is a facet of $M$ with $l$ vertices $v_{i_{1}}, v_{i_{2}}, \ldots, v_{i_{1}}$, then $\sum_{j=1}^{l} \operatorname{deg} v_{i_{1}} \leq F+2 l-1$.

\section{Proof}

Let $M$ be a w.n.p. map of genus $1 \frac{1}{2}$ with $V$ vertices. By adding diagonals, if necessary, $M$ gives rise to a polyhedral triangulated map of the same genus $1 \frac{1}{2}$ and with the same number $V$ of vertices. Using Euler's equation, a direct computation yields $V \geq 8$. Using more sophisticated methods, Ringel [7] proved that a polyhedral triangulation of the 2 -manifold of genus $1 \frac{1}{2}$ must have at least nine vertices, hence $V \geq 9$. From [4, Theorem 8] we get for $V$ the upper bound 11 .

Since $9 \leq V \leq 11,(7)$ and (20) yield that the possible values for $k_{1}$ are just 5 and 6 , and for $V=9$ also $k_{1}=4$. With those restrictions on $V$ and $k_{1}$ it is easy to find all the candidates for $p$-vectors which are not in direct contradiction to (1), (2), and (6) (cf. [5, Section 2]). There are altogether 21 such $p$-vectors; 13 of these fail to satisfy (13). The remaining eight candidates for $p$-vectors are not in direct contradiction to any of the equalities and inequalities mentioned above, and are summarized in Table 1 . We will now examine them one by one to show that only three of them (nos. 1, 2, and 5) are realizable. We start with the nonrealizable cases.

In the process of examining a "p-vector" $p$ we often draw the w.n.p. map $M$ which is supposed to realize $p$, or a fragment of it. $M$ is drawn as a planar map with identification on the boundary edges and vertices. As each vertex must "see"

Table 1. The candidates for $p$-vectors of the nonorientable w.n.p. maps with Euler characteristic -1 .

\begin{tabular}{crrrrr}
\hline Case no. & $V$ & $p_{3}$ & $p_{4}$ & $p_{5}$ & $p_{6}$ \\
\hline 1 & 9 & 8 & 6 & & \\
2 & 9 & 11 & 3 & 1 & \\
3 & 10 & 4 & 6 & 2 & \\
4 & 10 & 7 & 3 & 3 & \\
5 & 10 & 10 & & 4 & 1 \\
6 & 10 & 6 & 6 & & \\
7 & 11 & 1 & 4 & 5 & \\
8 & 11 & 4 & 1 & 6 &
\end{tabular}


all the other $V-1$ vertices, the $V-1$ vertices which see a certain vertex must be distinct. We use the labels $0,1, \ldots, V-1$ to indicate such a set of $V$ distinct vertices, and the labels $a, b, \ldots$ to indicate vertices that must be identified with some vertices in the set $\{0,1, \ldots, V-1\}$. A broken line segment indicates a line segment which either exists, i.e., is an edge in $M$, or does not exist, i.e., is a diagonal of some facet in $\boldsymbol{M}$.

We also use here the following notation. $A_{a b c}$ means that it happens twice that $b$ is adjacent to both vertices $a$ and $c$ on the boundary of the configuration, and hence it is already completely surrounded by facets (see [1, Fig. 3(c)])-usually too few of them. $B_{a b}$ means that the edge $a b$ belongs to two facets (and therefore, if on the boundary of the configuration, it must appear twice). $C_{\alpha}$ denotes that the facets which contain the vertex $a$ either do not close to a disc, or they form a disc which is too small in the sense that it does not contain all the vertices of the map. $D_{a b}$ indicates that $a b$ is a diagonal in some facet (and usually an edge or a diagonal in another facet as well, which means a contradiction) and $E_{a b}$ simply means that $a b$ is (or must be) an edge.

Case no. 3. Here $V=10, p_{3}=4, p_{4}=6, p_{5}=2$. If there is such a map $M$, then (12) with $k_{j}=5$ implies that each pentagon shares an edge with the other pentagon and four quadrangles. Therefore, the two pentagons share an edge $a b$. Lemma 1 implies that $V_{3}=0$, as there are no 3 facets with a total of 15 vertices. It follows that each of the vertices $a, b$ is incident to at least two pentagons and two quadrangles. But this violates Lemma 1 for any valence of $a, b$, and case no. 3 is therefore not realizable.

Case no 4. Here $V=10, p_{3}=7, p_{4}=p_{5}=3$. Assume there is such a map $M$. Then (12) with $k_{j}=5$ implies that each pentagon is adjacent to the other two pentagons and to at least two quadrangles, hence to at most one triangle. Lemma 1 implies that if there is a 3-valent vertex in $M$, then the three pentagons meet at that vertex. Thus, $V_{3} \leq 1$.

Assume $V_{3}=1$. Then the configuration of the three pentagons is as depicted in Fig. 2(a). Each of the vertices 1, 4, 7 must see all the other vertices of $M$, and is therefore adjacent either to three triangles or to a quadrangle and a triangle. Since $p_{3}=7$, at least one of these three vertices is adjacent to a quadrangle. Thus, by the symmetry, we may assume that 67 is an edge of a quadrangle. As each pentagon is adjacent to at most one triangle, we get that the configuration depicted in Fig. 2(a) is a part of the map $M$, and four triangles of $M$ are missing here. Clearly $\{a, b\}=\{2,3\},\{c, d\}=\{8,9\}$, and $\{e, f\}=\{5,6\}$.

If $a=2$, then $b=3, D_{26} \Rightarrow e=5, f=6$, and $D_{59} \Rightarrow c=8, d=9$. Considering the neighborhood of 3 , we see that two of the four missing triangles must be 356 and 359, but then the neighborhood of 6 is not a disc.

Hence $a=3, b=2 ; E_{38} \Rightarrow c=9, d=8 ; E_{59} \Rightarrow e=6, f=5$. But now the three facets incident to the vertex 2 already form a disc, without 2 seeing the vertices $5,8,9$. Thus $V_{3}=1$ is impossible.

Therefore, $V_{3}=0$. The three pentagons do not meet at a vertex. Hence there are six vertices each of which is incident to two pentagons and either to three 


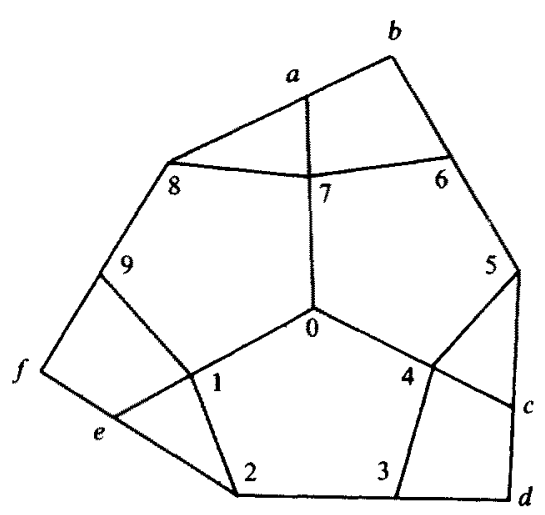

(a)

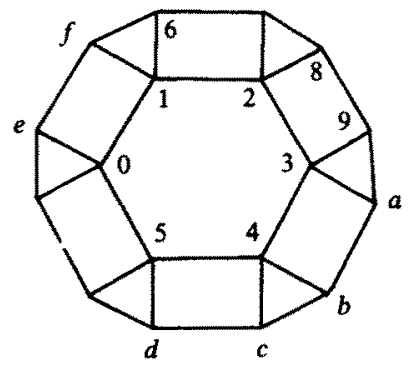

(b)

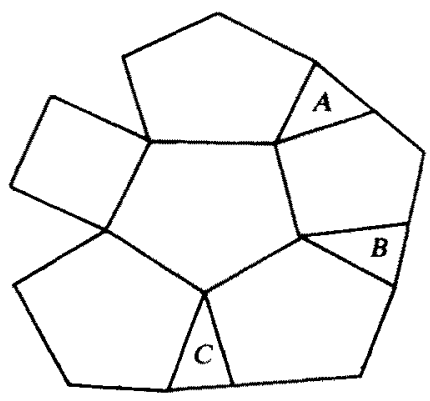

(c)

Fig. 2

triangles or to a quadrangle and a triangle. The configuration of the three pentagons and the fact that the elements of $M$ form a complex causes all these triangles and quadrangles to be distinct from each other. On the other hand, they cannot be distinct as their total number exceeds $p_{3}+p_{4}=10$.

Case no. 4 is therefore not realizable.

Case no 6. Here $V=10, p_{3}=p_{4}=6, p_{6}=1$. Inequality (12) (with $j=1$, i.e., $k_{j}=6$ ) implies that the hexagon shares an edge with each of the six quadrangles, and the map $M$, if exists, must be as depicted in Fig. 2 (b). Clearly, $\{a, b\}=\{6,7\}$ (as $a, b, 8,9$ see the vertex 3 ), hence $\{c, d\}=\{8,9\}$ and similarly $\{e, f\}=\{8,9\}$. Now the edge 89 appears three times-a contradiction.

Case no 7. Here $V=11, p_{3}=1, p_{4}=4, p_{5}=5$. Inequality (12) implies that each pentagon is adjacent to all the other four pentagons and to a quadrangle. This implies the existence of at least three triangles $(A, B, C$ in Fig. 2(c)), contradicting $p_{3}=1$.

Case no 8. Here $V=11, p_{3}=4, p_{4}=1, p_{5}=6$. Inequality (12) implies that each pentagon is adjacent either to four pentagons and a quadrangle or to five 
pentagons. As $p_{4}=1, p_{5}=6$, there is at least one pentagon adjacent to the five other pentagons. As in the previous case, this implies the existence of at least five distinct triangles, contradicting $p_{3}{ }^{\circ}=4$.

Next we turn to the three realizable cases.

Case no 1 . Here $V=9, p_{3}=8, p_{4}=6$. Let $M$ be such a map. Lemma 1 implies that $V_{3}=0$ and that

(*) for each $0 \leq i \leq 4$, a vertex of valence $4+i$ belongs to $4-i$ quadrangles and $2 i$ triangles.

Also, (10) implies that

(**) every quadrangle in $M$ is adjacent to at least two quadrangles.

We carry out our investigation in three steps. In steps I and II we show that $V_{8}=V_{5}=0$. We already know that $V_{3}=0$. Clearly $\sum_{i} V_{i}=9$, and (25) implies that $\sum_{i}(i-3) V_{i}=21$. Solving the set of these equations we see that there are just three solutions:

$$
\begin{aligned}
& \text { (a) } V_{4}=3, \quad V_{6}=6, \quad V_{7}=0 \text {, } \\
& \text { (b) } V_{4}=4, \quad V_{6}=3, \quad V_{7}=2 \text {, } \\
& \text { (c) } \quad V_{4}=5, \quad V_{6}=0, \quad V_{7}=4 \text {. }
\end{aligned}
$$

Now (b) and (c) are in direct contradiction to (16), and we are left with just one possibility, namely, $V_{4}=3, V_{6}=6$. Finally, in step III we show that this $v$-vector is indeed realizable in a unique way.

Step I. $V_{8}=0$

(*) for $i=4$ together with $p_{3}=8$ implies that $V_{8} \leq 1$. If there is in $M$ an 8-valent vertex $x$, then it is joined to all the other vertices and is incident to all the triangles in $M$. Thus each of the other eight vertices is incident to just two triangles, and is therefore (by (*)) of valence 5 . Hence $V_{5}=8, V_{8}=1$. But this contradicts (17), $v_{j}$ being the vertex $x$. Thus $V_{8}=0$.

Step II. $V_{5}=0$

Assume $V_{5}>0$. A 5 -valent vertex in $M$ is either of type 1, depicted in Fig. $3(\mathrm{a})$, namely, the two triangles incident to the 5 -valent vertex 0 share a common edge, or of type 2, depicted in Fig. 3(b), namely, these two triangles are not adjacent.

Assume there is in $M$ a 5-valent vertex 0 of type 1, and let its neighboring vertices be labeled as in Fig. 3(a). Figure 3(c) depicts a larger fragment of the map $M$. Here the dotted lines are edges or diagonals, $k$ is omitted if $d 1$ or $h 7$ are diagonals, and $k$ and $j$ are omitted if $d 1$ and $h 7$ are diagonals.

Note that the pair of facets adjacent to edge 17 (or the facet with 17 as diagonal) occurs twice in the figure. Clearly $\{a, b, c, d\}=\{4,5,6,7\}, d \neq 7$ and $\{e, f, g, h\}=\{1,2,3,4\}, h \neq 1$. 


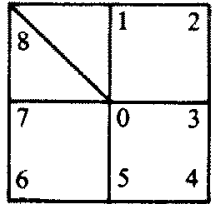

(a)

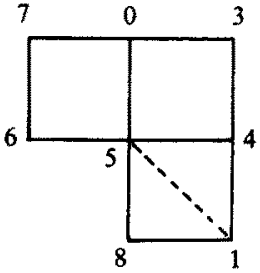

(d)

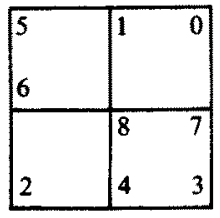

(g)

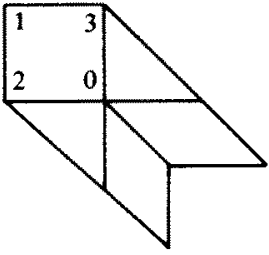

(b)

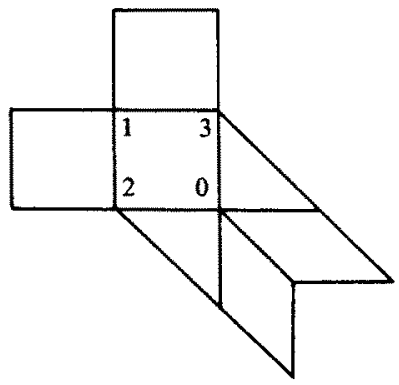

(e)

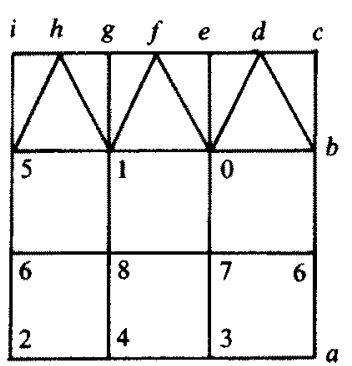

(h)

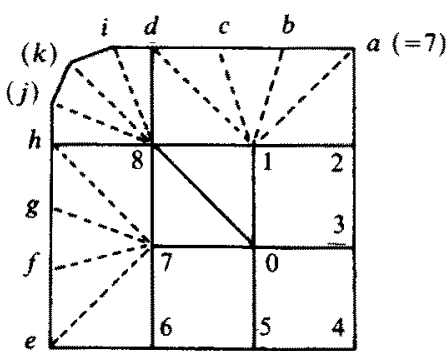

(c)

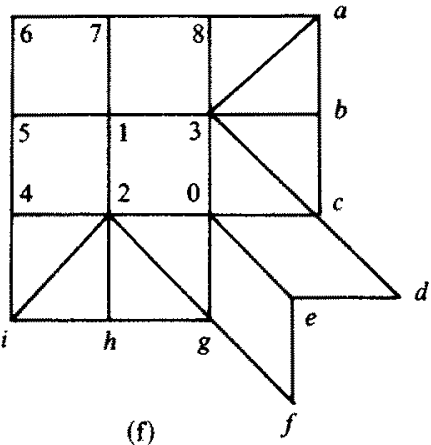

(f)

Fig. 3

If $7 \in\{b, c\}$ and $1 \in\{f, g\}$, we get a contradiction since $\{a, b, c, d\} \cap\{e, f, g, h\}=$ $\{4\}$ and the two neighbors of 1 (resp. 7) on the boundary of the figure have to coincide. The cases with $e=1$ are symmetric to the cases with $a=7$; therefore, we assume from now on, without loss of generality, that $a=7$.

If $e=1$, then $b=6, f=2$ hence $4 \in\{c, d\} \cap\{g, h\} .4=d=h$ is impossible since 8 sees $d$ and $h$. Without loss of generality (symmetry!) we may assume $e=4$, thus $d=5$. If $g=3, h=4$, we get the contradiction $A_{234}$ (note that $f 7=27$ is an 
edge). Thus $g=4, h=3$. Regarding parts of the stars of 3 and 5 , which are already determined, yields Fig. $3(\mathrm{~d})$. ( $c 1=41$ cannot be diagonal, since this would imply $D_{56}$, while $E_{56}$.) This implies that either the triangles 256 and 258 or the quadrangle 2658 are in $M$. By symmetry $((35)(26)(17))$ we get that either 632 and 638 or 6238 are in $M$. Combining the two we see, by considering the diagonals of these two quadrangles, that none of these quadrangles is possible. Thus we get the four triangles. Together with the triangles $018,078,127$ (since 26 is not a diagonal), 167 and 146 (since 56 is not a diagonal) we have more than eight triangles in $M$, which is a contradiction.

Thus $e \neq 1$. Therefore $f=1$ or $g=1$, and the two neighbors of $f$ (resp. $g$ ) on the boundary of Fig. 3(c) coincide with $b$ and 2 . Since $\{a, b, c, d\} \cap\{e, f, g, h\}=\{4\}$, we get $b=4$. Thus $\{c, d\}=\{5,6\}, c=5, d=6$ is impossible (since we would get $A_{456}$ if $b 1$ is an edge, otherwise the diagonal 57 would occur twice). Thus $c=6$, $d=5$. Furthermore $c 1$ and $b 1$ are edges (since $E_{45}, E_{67}$ ). Regarding the part of the star of 5 which is already determined, we get that either the two triangles 258,254 or the quadrangle 2854 are in $M$. In particular, 24 is an edge and hence $a 1$ is also an edge.

If the triangles 258,254 are in $M$ (which implies $i=2$ ), then $d 1$ is not a diagonal (otherwise the quadrangle $1 c d 8$ is adjacent to three triangles, in contradiction with $(* *)$ ) and the map $M$ contains more than eight triangles (namely, $258,254,158,156,164,147,127,108,078$ ), a contradiction. Hence the quadrangle 2854 is in $M$ and therefore $i=4$.

Recall that $1 \in\{f, g\}$. If $g=1$, then (as $\{h, f\}=\{2,4\}$ ) $h=2, f=4, e=3$, and $j$ and $k$ have to be canceled. Regarding the star of 3 we see that the quadrangle 2368 must be in $M$. But now the edge 28 appears three times (namely, in 2368, 287, and 2854), a contradiction. Therefore $f=1$. Thus $h=3 . e 7,17$, and $g 7$ are edges, since 16 and 24 are edges and 13 is a diagonal. Also, as $g \in\{2,4\}, E_{28}$ and $D_{48}$, we get that $h 7$ is an edge. Now $d 1$ must be a diagonal (otherwise we have more than eight triangles), hence $k$ has to be omitted and $j=2$. But now hj8 $(=238$ ) is a triangle in $M$, yielding a total of nine triangles in $M$, a contradiction.

Thus we conclude that $M$ does not contain any 5-valent vertex of type 1 .

If $M$ contains a 5-valent vertex 0 of type 2 , let the neighborhood of 0 be labeled as depicted in Fig. 3(b). By (**), the quadrangle 0213 is adjacent to at least two quadrangles, and we are led to the larger fragment of $M$ shown in Fig. $3(\mathrm{e})$. Now, by $(*)$, the valence of the vertex 1 is either 4 or 5 . If it is 5 , then the vertex 1 is of type 1 , which, as shown above, cannot exist in $M$. Hence the valence of 1 is 4 , and we may add an additional quadrangle incident to the vertex 1 , yielding a fragment of $M$ which contains two triangles and all six distinct quadrangles. It follows that all the facets of $M$ incident to 2 or 3 that are not in this fragment are triangles. Thus, 2 and 3 are of valence 6, and Fig. 3(f) therefore depicts the entire map $M$.

Here $\{a, b, c\}=\{4,5,6\},\{g, h, i\}=\{6,7,8\},\{c, d, e, f, g\}=\{4,5,6,7,8\}$. Clearly $b \neq 5$ (as otherwise $A_{456}$ ) and similarly $h \neq 7$.

Assume $a=5$. If $b=4$, then $c=6$, hence $e \notin\{4,5,7\}$ (as $\left.E_{46}, E_{56}, E_{76}\right)$; therefore $e=8$. This implies $g \notin\{5,6,7\}$, but $g \neq 4$ too, as there is already an edge 24 . Thus $b=4$ is impossible. Hence $b=6$. This implies $c=4$ which implies $e \neq i$. Obviously 
$e \notin\{g, h\}$. Thus $e \notin\{6,7,8\}$, hence $e=5$, but this is not possible as 45 is now both an edge and a diagonal.

Thus $a \neq 5$, and, because of the symmetry, also $i \neq 7$. Therefore, $c=5$ and $g=7$. Now $D_{e s}$ and $D_{e 7}$ imply that $e \notin\{4,6,8\}$, a contradiction.

This concludes step II, showing that $V_{5}=0$.

As already stated, this leaves us with just one candidate for a $v$-vector, namely, $V_{4}=3, V_{6}=6$.

Step III. There is a unique map $M$ satisfying $V_{4}=3, V_{6}=6$.

Let 8 be a 4-valent vertex, and take the labeling of the neighborhood of 8 to be as depicted in Fig. $3(\mathrm{~g})$. Lemma 2 implies that there are no three 6-valent vertices in the same quadrangle; thus we may assume without loss of generality that the six 6-valent vertices of $M$ are $0,1,2,3,4,5$, while $6,7,8$ are 4-valent. This implies that the configuration of Fig. 3(h) is a part of $M$ (possibly the entire map $M$ ), and $\{a, b\}=\{2,5\}$. All the facets here, except for the triangle $b c d$, touch the facet 1780 and are therefore distinct from each other. A priori it is not clear whether the triangles $b c d$, ih 5 are distinct from the other triangles.

If $a=2$, then $b=5$; hence $\{h, g, f\}=\{f, e ; d\}=\{2,4,3\}$. As $h \neq f \neq d$ and $g \neq e$, it follows that the sequence $d, e, f, g, h$ contains the consecutive triple $2,4,3$ (perhaps in reversed order), thus yielding the contradiction $C_{4}$.

Hence $a=5, b=2$. Clearly $\{d, e, f\}=\{3,4,5\},\{f, g, h\}=\{2,3,4\}$ and $\{c, d\} \subset$ $\{1,3,5\}$. Thus $d \in\{3,5\}$ and $f \in\{3,4\}$. Obviously $e \neq 3$ (otherwise $A_{435}$ ) and $g \neq 4$ (otherwise $A_{243}$ ).

Now, if $d=5$, then $e=4, f=3, g=2, h=4$, and considering the neighborhood of 2 we get $c=3$. This is indeed a w.n.p. map which satisfies all the requirements, and is exactly the map $A$ depicted in Fig. 1 .

If, on the other hand, we take $d=3$, then $e=5, f=4, e=5 \Rightarrow c \neq 5 \Rightarrow c=1$. $B_{35} \Rightarrow h \neq 3 \Rightarrow h=2 \Rightarrow g=3$. In order to get from this the desired w.n.p. map we should cancel one occurrence of the triangle 123, and add the triangle 245. However, this w.n.p. map is isomorphic to the previous one under the permutation $((04)(13)(78))$.

Case no. 2. Here $V=9, p_{3}=11, p_{4}=3, p_{5}=1$. It follows from (10) that the pentagon is adjacent to each of the three quadrangles (and touches all the facets of the map). The three edges of adjacency may be consecutive (Fig. 4(a)) or not (Fig. 4(b)).

In the first case, assume the labeling as in Fig. $4(a)$. Since $a, b$ see the vertex 4, we have $\{a, b\}=\{5,6\}$. As $\{a, b, c, d\}=\{5,6,7,8\}$, this implies $\{c, d\}=\{7,8\}$. But we also have $\{g, h\}=\{7,8\}$ (as $g, h$ see 1 ), and so we have the edge 78 three times, a contradiction.

Thus our map $M$ must be as depicted in Fig. 4(b). As before we have $\{a, b\}=\{5,6\},\{g, h\}=\{7,8\}, c \in\{7,8\}, d \notin\{7,8\}$ (otherwise 78 appears three times), hence $d=a$. The symmetry of the map implies $e=h$. Since $f \notin\{d, e, g\}$, we have $f=b$, and by the symmetry $c=g$.

Now, if $a=5$, then $f=b=6, h \neq 8$ (otherwise 58 appears three times) hence $h=e=7, g=c=8$. This is indeed a w.n.p. map satisfying the requirements, the map $\mathscr{B}$ depicted in Fig. 1. 


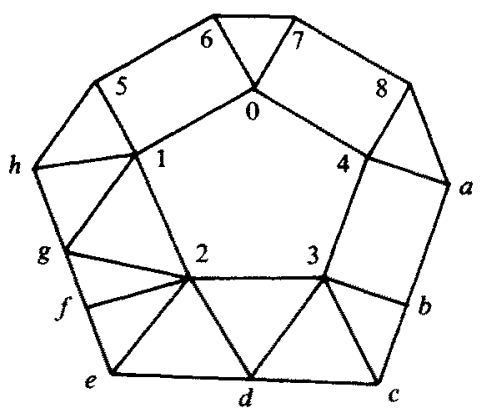

(a)

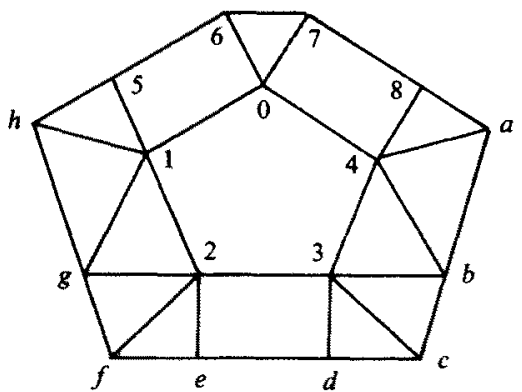

(b)

Fig. 4

If, on the other hand, we take $a=6$, then $b=5$. Now there are two possibilities: either $g=7, h=8$ and we do indeed get a w.n.p. map as required-but this map is isomorphic to the previous one under $(14)(23)(58)(67)$-or $g=8, h=7$, and we get the w.n.p. map $\mathscr{C}$ shown in Fig. 1, which is not isomorphic to the previous map. Thus case no. 2 has two distinct realizations.

Case no. 5. $V=10, p_{3}=10, p_{5}=4$. There are no four facets with a total of 17 vertices, hence Lemma 1 implies that $V_{4}=0$. Similarly, $V_{6}=V_{8}=0$. Thus, (25) implies $V_{5}+2 V_{7}+3 V_{9}=10$ and obviously $\sum V_{i}=V_{3}+V_{5}+V_{7}+V_{9}=10$, and these yield $V_{3}=V_{7}+2 V_{9}$. It follows that either $V_{5}=10$ or $V_{3}>0$

If $V_{5}=10$, then, by Lemma 1 each vertex of $M$ is incident to two pentagons and three triangles. It follows (as $p_{5}=4$ ) that each pentagon is adjacent to two of the other pentagons and has a unique common vertex with the remaining pentagon. Thus each pentagon (see Fig. $5(\mathrm{a})$ ) touches 12 triangles which, as they all touch the same pentagon, must be all distinct. But this is impossible, as $p_{3}=10$.

Thus $V_{3}>0$. Let 0 be a 3 -valent vertex in $M$, and let $01,04,07$ be the edges incident to it. Lemma 1 implies that a 3-valent vertex in $M$ is incident to just three pentagons. If none of the vertices $1,4,7$ is incident to a third pentagon, then each of these vertices is of valence 5 , and the configuration depicted in Fig. $5(\mathrm{~b})$ is contained in $M$. As all ten vertices of the map $M$ are in the three pentagons incident to 0 (see Fig. 5(b)), the fourth pentagon must be incident to two of the "free" edges $23,56,89$ and to one of the remaining two "free" vertices. Thus we may assume, without loss of generality, that the edge 23 belongs to the fourth pentagon. But, on the other hand, $\{a, b\}=\{2,3\}$, so the edge 23 is incident to three facets, a contradiction.

It follows that one of the vertices $1,4,7$-say 1 -is incident to three pentagons. This leads to Fig. $5(\mathrm{c})$. (Since $\{a, b\}=\{5,6\}, 8$ is incident to just one pentagon, hence to six triangles.) Here all the facets are distinct, as they all touch the pentagon 01987, and therefore this is the entire map $M$. We have $\{a, b\}=\{5,6\}$, $\{c, d\}=\{3,4\},\{i, h\}=\{2,3\}$, and $\{d, e, f, g, h\}=\{2,3,4,5,6\}$. 


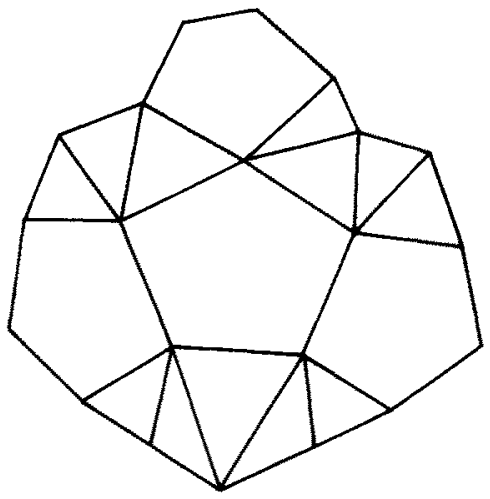

(a)

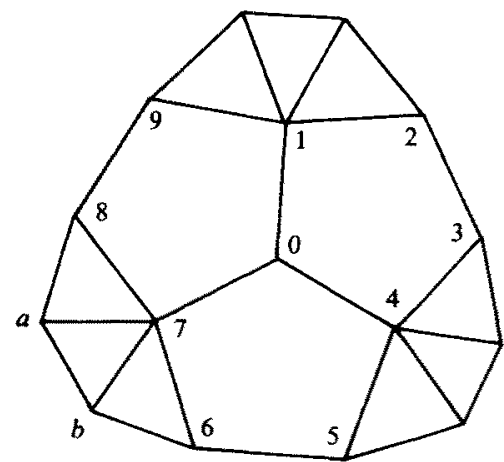

(b)

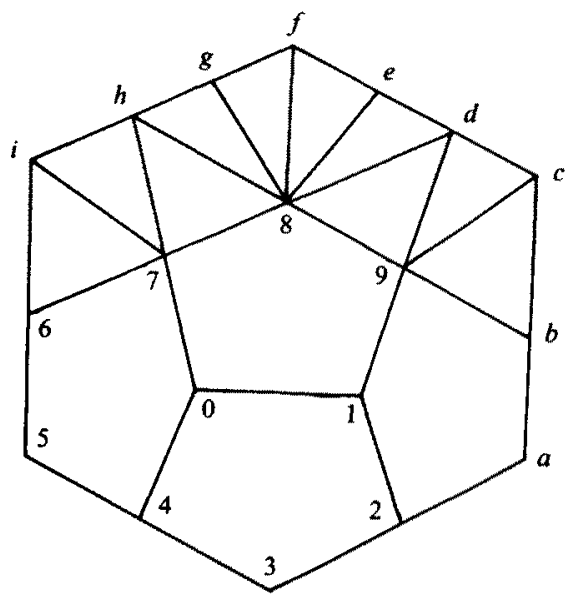

(c)

Fig. 5

Assume $a=5$. Then $b=6, c \neq 4\left(D_{46}\right) \Rightarrow c=3 \Rightarrow d=4$. Also $b=6 \Rightarrow i \neq 2\left(D_{26}\right) \Rightarrow$ $i=3$. Now we have the contradiction $A_{365}$.

Hence $a=6, b=5$. If $c=4$, then $d=3$ and we have the contradiction $A_{543}$, hence $c=3, d=4$. Similarly, $i=3, h=2$. Now $\{e, f, g\}=\{3,5,6\}$, hence $f=3$ (otherwise edge 56 appears three times). Finally, $D_{25} \Rightarrow g=6, e=5$, and we get the map $\mathscr{D}$ depicted in Fig. 1. This is indeed a w.n.p. map as required.

This concludes the investigation of case no. 5, and also concludes the proof of our theorem.

\section{Alternative Construction}

In the following we describe an alternative way to obtain the four w.n.p. maps of genus $1 \frac{1}{2}$ (i.e., Euler characteristics -1 ). The advantage of this alternative 
process is its elegance and simplicity: we obtain the four maps $\mathscr{A}, \mathscr{A}, \mathscr{C}, \mathscr{D}$ from four known w.n.p. maps A, B, C, D of lower genus by a simple replacement operation, which is of a general nature. Its main disadvantage is that a priori it is not clear that this process yields all the w.n.p. maps of genus $1 \frac{1}{2}$. We use here two replacement operations (for more general treatment see [3]):

Replacement by a Möbius strip. Let $T_{1}$ be the triangulated Möbius strip depicted in Fig. 6. Note that $T_{1}$ is weakly neighborly and has a pentagonal boundary $a, b, \ldots, e$ which contains all five vertices of $T_{1}$. If $M$ is any w.n.p. map which contains a pentagonal facet $F$, then the removal of $F$ yields a w.n.p. map with a pentagonal boundary and the glueing of $T_{1}$ to this map done by identification of their boundaries yields a new map $M_{1}$. It is clear that $M_{1}$ is a w.n.p. map, has the same number of vertices as $M$, is not orientable, and its genus is greater by $\frac{1}{2}$ than that of $M$. We say that $M_{1}$ is obtained from $M$ by replacing the facet $F$ of $M$ by the Möbius strip $T_{1}$.

Replacement by Császár's torus. Let $T_{2}$ be the triangulated torus with boundary depicted in Fig. 6. $T_{2}$ is obtained from Császár's torus (i.e., the map $\mathscr{A}$ in [5], see also [6]) by removing the star of one of its seven vertices. Thus $T_{2}$ is a w.n.p.

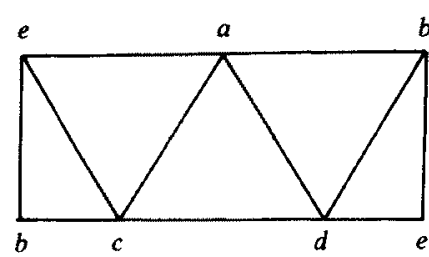

$T_{1}$

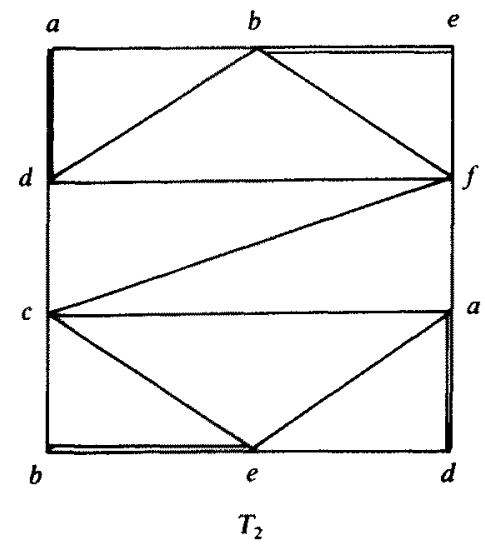

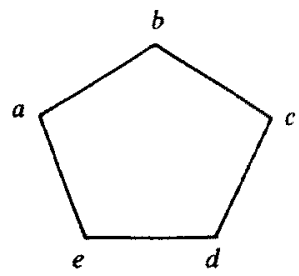

bd $T_{1}$

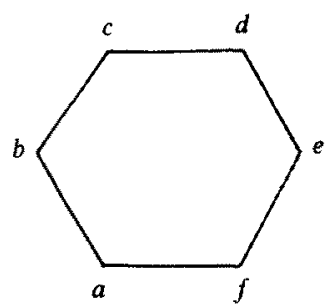

bd $T_{2}$

Fig. 6. Möbius strip with pentagonal boundary and truncated Császár's torus. 
map on the torus with an hexagonal boundary $a, b, \ldots, f$ and all its six vertices are on the boundary. If $M$ is any w.n.p. map which contains an hexagonal facet $F$, then the map $M_{2}$ obtained from $M$ by replacing the facet $F$ of $M$ by (truncated) Császár's torus $T_{2}$ is defined in analogy to the previous case. $M_{2}$ is a w.n.p. map with the same number of vertices as $M$, its genus is greater by 1 than that $M$, and it preserves the orientability of $M$, that is, $M_{2}$ is orientable if and only if $M$ is orientable.

Obviously, these two replacement operations can be repeatedly applied to the same w.n.p. map $M$ as many times as the number of pentagonal and hexagonal facets permits.

Now Fig. 7 depicts four w.n.p. maps A, B, C, D. A, C, and D are projective planes $\left(g=\frac{1}{2}\right)$ with 9,9 , and 10 vertices, respectively (see [3]) and $B$ is a torus

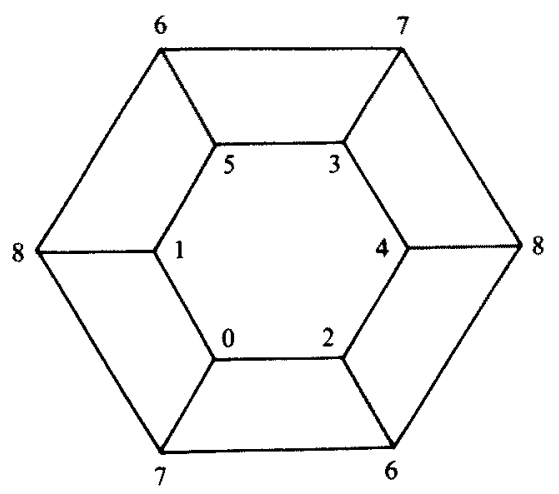

A

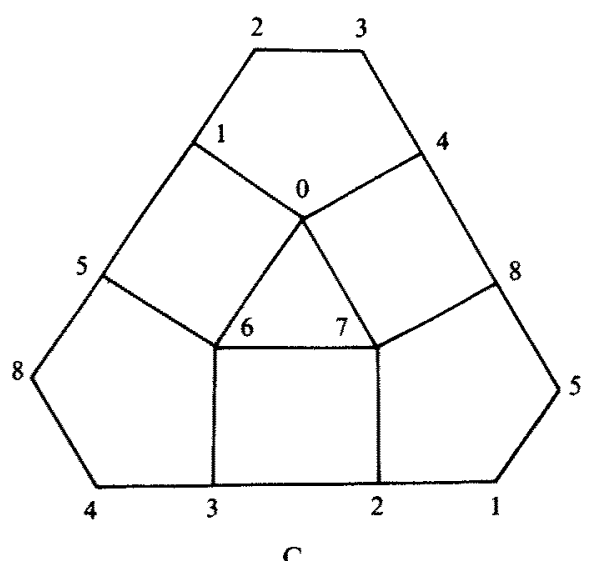

C

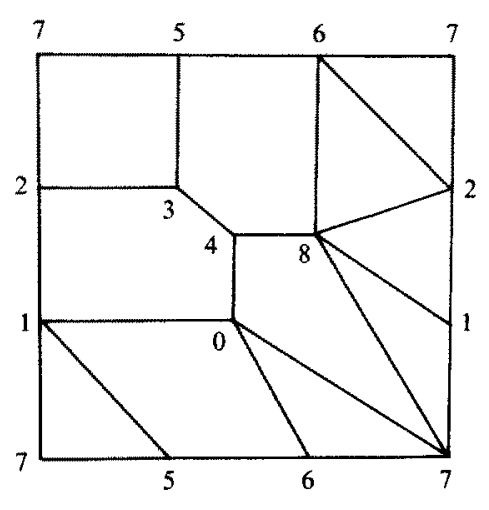

B

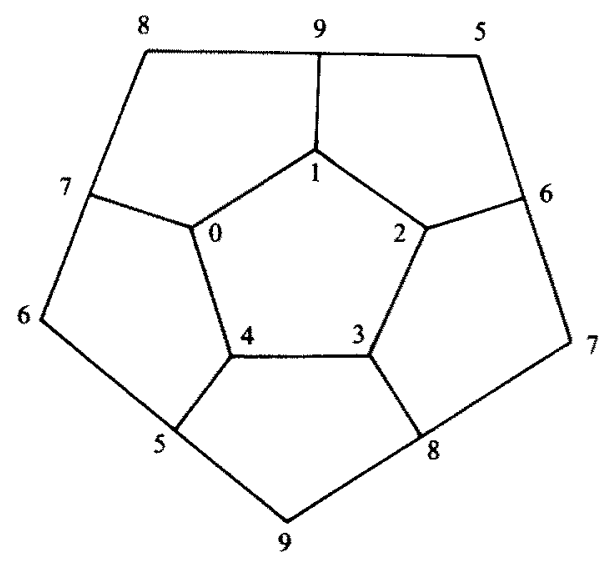

D

Fig. 7 
( $g=1$ ) with 9 vertices (see [5]). Map $\mathscr{A}$ is obtained from map A by replacing the hexagon 024351 by Császár's torus $T_{2} ; \mathscr{B}$ is obtained from map B by replacing the pentagon 34865 by the Möbius strip $T_{1} ; \mathscr{C}$ is obtained from map $\mathrm{C}$ by repeatedly applying the Möbius strip replacement operation at the pentagon 12785 and at the pentagon 34856, and map $\mathscr{D}$ is obtained from map D by a similar application of the Mobius strip replacement operation at the pentagons 26783 and 34598.

Symmetry groups. The symmetry group of map $\mathscr{A}$ is the cyclic group $Z_{6}$, generated by $(510243)(687)$ (the symmetry group of map $A$ is the dihedral group $D_{6}$, but it reduces by the replacement operation, as the symmetry group of the truncated Császár torus is only $\left.Z_{6}\right)$. Map $\mathscr{B}$ is asymmetric. Map $\mathscr{C}$ has $Z_{2}$ generated by (14)(23)(58)(67) as its symmetry group and the group of map $\mathscr{D}$ is $Z_{2} \times Z_{2}$, with generators $(01)(24)(56)(79)$ and $(29)(38)(47)(56)$.

\section{References}

1. A. Altshuler and U. Brehm, Non-existence of weakly neighborly polyhedral maps on the orientable 2-manifold of genus $2, J$. Combin. Theory Ser. A, to appear.

2. A. Altshuler and $U$. Brehm. The weakly neighborly polyhedral maps on the non-orientable 2-manifold with Euler characteristic -2, to appear.

3. U. Brehm, Weakly neighborly polyhedral maps on the projective plane, the Möbius strip and the Klein bottle, to appear.

4. U. Brehm and A. Altshuler, On weakly neighborly polyhedral maps of arbitrary genus, Israel $J$. Math. 53 (1986), 137-157.

5. U. Brehm and A. Altshuler, The weakly neighborly polyhedral maps on the torus, Geom. Dedicata 18 (1985), 227-238.

6. A. Császár, A polyhedron without diagonals, Acta Sci. Math. (Szeged) 13 (1949-50), 140-142.

7. G. Ringel, Wie man die geschlossenen nichtorientierbaren Flächen in möglichst wenig Dreiecke zerlegen kann, Math. Ann. 130 (1955), 317-326.

Received April 30, 1985. 